\title{
Evaluation of a Pediatric Mock Code Educational Training Program at a Large, Tertiary Care Pediatric Hospital
}

\author{
Ayelet Rimon ${ }^{1,2}$, Amit Hess ${ }^{1,2 *}$, Dennis Scolnik ${ }^{3}$, Oren Tavor ${ }^{1,2}$, Shirley Friedman ${ }^{2,4}$, \\ Miguel Glatstein ${ }^{1,2 \#}$ \\ ${ }^{1}$ Pediatric Emergency Medicine, Dana-Dwek Children's Hospital, Tel-Aviv, Israel \\ ${ }^{2}$ Sackler School of Medicine, Tel Aviv University, Tel-Aviv, Israel \\ ${ }^{3}$ Divisions of Pediatric Emergency Medicine and Clinical Pharmacology and Toxicology, Department of \\ Pediatrics, The Hospital for Sick Children, University of Toronto, Toronto, Canada \\ ${ }^{4}$ Pediatric Intensive Care, Dana-Dwek Children's Hospital, Tel-Aviv, Israel \\ Email: "Nopasara73@hotmail.com
}

Received 18 October 2015; accepted 14 December 2015; published 17 December 2015

Copyright (C) 2015 by authors and Scientific Research Publishing Inc.

This work is licensed under the Creative Commons Attribution International License (CC BY).

http://creativecommons.org/licenses/by/4.0/

cC) (7) Open Access

\begin{abstract}
Background: Management of the acutely ill children represents one of the more complex clinical skills required of pediatric physicians. Our goal was to develop and evaluate a multidisciplinary pediatric mock code training program for the pediatric residents in our institution. Methods: We performed a before and after evaluation of pediatric residents. The residents were educated by attending five mock code scenarios, followed by debriefing. Before and after the five sessions, the residents completed a self-assessment questionnaire. Results: Residents reported a significant improvement in their comfort in all aspects of managing pediatric resuscitations, with notable improvement seen in running a resuscitation requiring airway management, managing fluid resuscitation and performing endotracheal intubation. The most prominent change was demonstrated in the comfort level of the overall management of a pediatric resuscitation. Conclusion: The pediatric mock code educational training program improved residents' self-reported knowledge and comfort level in managing pediatric emergency situations.
\end{abstract}

\section{Keywords}

Education, Mock Code, Pediatric Residents, Emergency Medicine

\footnotetext{
*The authors have the equal contribution.

"Corresponding author.

How to cite this paper: Rimon, A., Hess, A., Scolnik, D., Tavor, O., Friedman, S. and Glatstein, M. (2015) Evaluation of a Pediatric Mock Code Educational Training Program at a Large, Tertiary Care Pediatric Hospital. Open Journal of Pediatrics, 5, 314-319. http://dx.doi.org/10.4236/ojped.2015.54047
} 


\section{Background}

Management of acutely ill children represents one of the more complex clinical skills required of physicians, especially in the pediatric emergency department where severe medical conditions, behavioral crises, and accidental/intentional injuries are commonly encountered [1] [2]. The ability of the physician to competently and effectively manage these varied medical conditions depends both on their total caseload and the frequency of exposure to these types of injuries and situations [3]. Although pediatric residents are expected to acquire this set of skills during their training since they are often the primary care givers during resuscitations, their degree of confidence and knowledge levels in managing these situations remains unclear [4]. Critical decisions regarding very ill children presenting to the emergency department often have to be taken by pediatric residents before the arrival of the sub-specialties such as anesthesia, general surgery and pediatric critical care. Teaching interventions have been designed to address residents' needs in these areas. PALS is also evidence based and a standard of care, although skills retention beyond 6 months decreases. Although the Pediatric Advanced Life Support (PALS) course has been shown to be an effective teaching program, it has several limitations including extensive facility and faculty requirements, high cost and limited availability [5]. PALS is also evidence based and a standard of care, although skills retention beyond 6 months decreases. Mock code training is an effective training tool for pediatric acute care providers who have limited exposure to critically ill patients [6] [7], and pediatric "mock codes" have been utilized to increase the emergency preparedness of inpatient medical units for several decades [8]. Repetitive pediatric simulation of this type provides learners with a discrete opportunity to apply their knowledge [9]. These practice drills have been shown to both increase practitioners' confidence and decrease anxiety during actual resuscitations [2]. Data obtained from medical trainees can also be used to design future skill-based educational initiatives [5].

The objective of this educational study was to improve medical caregivers' skills in pediatric resuscitation at our institution. The low fidelity simulation training was administered in the actual emergency department in order to provide pediatric residents with as real an experience as possible and to facilitate their transition into becoming a part of the pediatric emergency team. We hypothesized that weekly training, comprised of multiple mock code scenarios, for two months would increase pediatric residents' reported levels of knowledge and comfort in leading a real code.

\section{Methods}

Over a period of two months (from April 1, 2014 to May 31, 2014), all junior and senior residents in the pediatric residency program of our institution were invited voluntary to attend weekly mock code teaching and debriefing sessions. Prior to beginning the course of mock code educational sessions, residents completed a self-assessment questionnaire (pre). Questions regarding their comfort in dealing with emergency situations and confidence in performing specific life-saving skills were addressed, using a five-point Likert scale ranging from 1 = "strongly disagree" to 5 = "strongly agree" for each item (see Appendix 1). Once the training was completed each resident completed the same self-assessment questionnaire (post) to measure changes resulting from the intervention. Data was also recorded regarding the number of occasions each resident had participated in emergency interventions or resuscitations before starting the training program.

The intention of each mock code scenario was to provide specific opportunities for the residents to care for seriously ill pediatric patients. Participants were exposed to situations that required immediate attention to the "ABCs" (airway, breathing and circulation) of pediatric resuscitation using a traditional plastic mannequin (Laerdal Medical Corporation, USA). The transition from basic to advanced life support techniques was inherent in each mock code scenario. We hoped to bring all residents to a self-assessed knowledge and skill level of at least "average" (2.5/5) in each skill assessed and also aimed to eliminate responses indicating the lowest levels of confidence (strongly disagree or disagree with having good resuscitation knowledge and skills). In order to create a realistic simulation, nurses were included in the mock code training and the training took place in the resuscitation room of the emergency department. The necessary equipment was present, but had been not set up or connected before the start of the scenario.

The 15 - 20 minute scenarios were designed and facilitated by emergency medicine physicians, and were followed by a 20 minute debriefing session. They were designed to be realistic and practical; life-threatening situations encompassing the wide range of childhood illnesses and injuries that practitioners may well have to address were specifically chosen. There were five different scenarios, all involving management of airway, 
breathing and circulation, due to different emergencies. Practical skills such as endotracheal intubation and obtaining intra-osseous access were incorporated in most scenarios.

Each of the five mock code scenarios was designed to encourage the development of a particular skill set: 1) Drowning: to familiarize residents with management priorities when dealing with drowning patients, recognize and treat hypothermia and its complications and also to consider potential associated problems like intoxication, head and cervical injury; 2) septic shock-meningococcemia: to enhance recognition of fever and petechiae as a medical emergency, to understand that systolic blood pressure can be preserved until late in the course of shock in children and to become cognizant that early and aggressive fluid resuscitation and vasopressor therapy are lifesaving; 3) altered mental status - ketoacidosis: to recognize and manage patients with altered mental status, identify the presenting signs and symptoms of diabetic ketoacidosis (DKA), to treat DKA and manage increased intracranial pressure associated with DKA; 4) apnea-bronchiolitis: to identify respiratory distress and impending respiratory failure, acutely manage apnea and respiratory failure, and to be aware of the association between apnea and bronchiolitis; 5) cardiac arrest: to rapidly start protocolized management of cardiac arrest and to acquire an initial approach to cardiac emergencies.

All codes were assessed for patient management and team function by an observing pediatric emergency medicine attending physician. At the completion of each scenario, the participating residents took part in a debriefing session with the attending physician, which pointed out the strengths and weaknesses of the team.

Statistical Analysis: In addition to using descriptive statistics to describe findings data from the pre and post self assessment questionnaire responses were compared using the Wilcoxon Signed Rank Test.

\section{Results}

Forty four pediatric residents participated in the pediatric mock code educational initiative, and completed both a pre and a post questionnaire. The group consisted of 33 (75\%) female residents, 11 males, ages 28 - 35 years. Six residents were unable to enter the training, as a result of leave of absence or rotations in a unit which refused nonattendance. Information regarding the characteristics of the participating residents is summarized in Table 1. The majority of the residents were junior, within the first two years of a five year residency. Twenty seven residents (61\%) had completed PALS training before the beginning of the training program. Previous experience in real resuscitations was variable: $36 \%$ of residents had no resuscitation experience, and $86 \%$ had been directly involved in five or less pediatric resuscitations.

Responses for all questions were calculated and reported as median scores before and after the mock code training (Table 2). Before the intervention the only skill the residents were comfortable performing was bag-mask-ventilation, with a median score of four (agree with the phrase "I am comfortable performing bagmask-ventilation”). Low scores were found on assessments of theoretical knowledge of airway management

Table 1. Demographic information of residents participating in the pediatric mock code educational initiative.

\begin{tabular}{cc}
\hline Characteristics of the 44 participants & $n(\%)$ \\
\hline Resident year of training & $12(27 \%)$ \\
\hline First (junior) & $14(32 \%)$ \\
Second (junior) & $5(11 \%)$ \\
Third (senior) & $7(16 \%)$ \\
Fourth (senior) & $6(14 \%)$ \\
Fifth (senior) & $12(27 \%)$ \\
Completed PICU rotation & $27(61 \%)$ \\
Completed PALS training & $16(36 \%)$ \\
\hline Actual resuscitation experience (number of cases) & $14(32 \%)$ \\
None & $8(18 \%)$ \\
\hline
\end{tabular}


Table 2. Summary of resident responses to the pre- and post-intervention questionnaire.

\begin{tabular}{|c|c|c|c|}
\hline Question & $\begin{array}{l}\text { Pre-intervention } \\
\text { (median, range) }\end{array}$ & $\begin{array}{l}\text { Post-intervention } \\
\text { (median, range) }\end{array}$ & p value \\
\hline I have good knowledge on how to manage a resuscitation requiring airway management & $2.5,5$ & 4,5 & $<0.001$ \\
\hline I feel comfortable running a resuscitation requiring airway management & 2,5 & 3,5 & $<0.001$ \\
\hline I have good knowledge on how to manage a resuscitation of shock requiring fluid resuscitation & 3,5 & 4,5 & $<0.001$ \\
\hline I feel comfortable running a resuscitation of shock requiring fluid resuscitation & 3,5 & 4,5 & $<0.001$ \\
\hline I am comfortable performing bag-mask-ventilation & 4,5 & 4.5, 5 & $<0.001$ \\
\hline I am comfortable performing endotracheal intubation & 2,5 & 4,5 & $<0.001$ \\
\hline I feel comfortable managing a pediatric resuscitation & 2,5 & $3.5,5$ & $<0.001$ \\
\hline
\end{tabular}

Table 3. Numbers of students scoring in lowest 2 categories pre- and post-mock code training.

\begin{tabular}{|c|c|c|c|}
\hline Question & $\begin{array}{l}\text { Bottom } 1+2 \text { score } \\
\text { pre-intervention, } n\end{array}$ & $\begin{array}{l}\text { Bottom } 1+2 \text { score } \\
\text { post-intervention, } n\end{array}$ & $\begin{array}{l}\text { Observed } \\
\text { change }\end{array}$ \\
\hline I have good knowledge on how to manage a resuscitation requiring airway management & 22 & 5 & 17 \\
\hline I feel comfortable running a resuscitation requiring airway management & 33 & 5 & 28 \\
\hline $\begin{array}{l}\text { I have good knowledge on how to manage a resuscitation of shock requiring fluid } \\
\text { resuscitation }\end{array}$ & 15 & 0 & 15 \\
\hline I feel comfortable running a resuscitation of shock requiring fluid resuscitation & 20 & 0 & 20 \\
\hline I am comfortable performing bag-mask-ventilation & 7 & 0 & 7 \\
\hline I am comfortable performing endotracheal intubation & 29 & 9 & 20 \\
\hline I feel comfortable managing a pediatric resuscitation & 33 & 2 & 31 \\
\hline
\end{tabular}

during resuscitation and fluid management in shock.

Numbers of residents who assessed themselves as being in the weakest categories of knowledge and resuscitation skills pre- and post-mock code training are displayed in Table 3 . The biggest change by the end of the program was in overall comfort level in the management of a pediatric resuscitation, with a decrease from 33 physicians in the bottom scores (1 or 2/5) pre-intervention (75\%) to two physicians (5\%) post-intervention. Notable improvement was also seen in running a resuscitation requiring airway management, fluid resuscitation for shock and performing endotracheal intubation. A small group of seven residents (16\%) reported having a low comfort level in bag-mask ventilation pre-intervention, in comparison to none in the post-intervention assessment.

\section{Discussion}

Pediatric residents are frequently the first responders in emergency situations in the emergency department even though they may have only modest experience as members of a pediatric resuscitation team and despite reporting low self-assessments of knowledge and skills of resuscitation. The "pre" results of our study clearly confirm the need for resuscitation training in the curriculum of residents. We have shown that a two-month simulation program involving five resuscitation scenarios can improve their self-assessment of knowledge and resuscitation skills.

The ability to lead resuscitation is one of the goals of training a pediatric resident. Traditionally, residents training in pediatrics gain much of their experience in resuscitation through exposure to real cases, with the only mandatory training being PALS. Despite the fact that our institution does not have the capability of high fidelity simulation, we demonstrated the after two months of intensive low fidelity weekly mock codes we were able to improve residents' knowledge and comfort level in managing emergency situations.

In a cross sectional study of pediatric residents Friedman $e t$ al. [8] showed that one year after starting a mock code program, residents attended more mock codes and reported more comfort with knowledge in codes. A second one year study also showed that mock codes improved resident confidence and self-assessment of their resuscitation skills [10]. Our study demonstrated an increase in residents' comfort managing pediatric resuscitations and performing procedural skills after an intensive two month training program. 
This study has several limitations. Firstly, given that the study was initiated due to an urgent need to train our residents, they served as their own control group through pre-post mock-code training. Secondly, the evaluation tool was resident self-assessment regarding knowledge and skills rather than a measure of real-life performance. Thirdly, previous studies have shown that participants in advanced life support courses have significant deterioration of their skills and knowledge over time [11] [12] and the sustainability of the observed improvements was not measured in our study. However, it may be reasonable to presume that residents' improvements will be reinforced by the real-life situations they deal with as they mature into fully-fledged pediatricians.

\section{Conclusion}

Our study stresses the need for mock code training in the curriculum of pediatric residents. It suggests a method whereby knowledge and skills can be developed and enhanced through the use of five common clinical scenarios, delivered on a weekly basis over two months, using low fidelity mock code training.

\section{References}

[1] Mikrogianakis, A., Osmond, M.H., Nuth, J.E., Shephard, A., Gaboury, I. and Jabbour, M. (2008) Evaluation of a Multidisciplinary Pediatric Mock Trauma Code Educational Initiative: A Pilot Study. The Journal of Trauma, 64, 761-767.

[2] Toback, S.L., Fiedor, M., Kilpela, B. and Reis, E.C. (2006) Impact of a Pediatric Primary Care Office-Based Mock Code Program on Physician and Staff Confidence to Perform Life-Saving Skills. Pediatric Emergency Care, 22, 415422. http://dx.doi.org/10.1097/01.pec.0000221342.11626.12

[3] Cappelle, C. and Paul, R.I. (1996) Educating Residents: The Effects of a Mock Code Program. Resuscitation, 31, 107-111. http://dx.doi.org/10.1016/0300-9572(95)00919-1

[4] Tofil, N.M., Lee White, M., Manzella, B., McGill, D. and Zinkan, L. (2009) Initiation of a Pediatric Mock Code Program at a Children's Hospital. Medical Teacher, 31, e241-e247. http://dx.doi.org/10.1080/01421590802637974

[5] Popp, J., Yochum, L., Spinella, P.C., Donahue, S. and Finck, C. (2012) Simulation Training for Surgical Residents in Pediatric Trauma Scenarios. Connecticut Medicine, 76, 159-162.

[6] Weinberg, E.R., Auerbach, M.A. and Shah, N.B. (2009) The Use of Simulation for Pediatric Training and Assessment. Current Opinion in Pediatrics, 21, 282-287. http://dx.doi.org/10.1097/MOP.0b013e32832b32dc

[7] Ralston, M.E., Day, L.T., Slusher, T.M., Musa, N.L. and Doss, H.S. (2013) Global Paediatric Advanced Life Support: Improving Child Survival in Limited-Resource Settings. Lancet, 381, 256-265. http://dx.doi.org/10.1016/S0140-6736(12)61191-X

[8] Friedman, D., Zaveri, P. and O’Connell, K. (2010) Pediatric Mock Code Curriculum: Improving Resident Resuscitations. Pediatric Emergency Care, 26, 490-494. http://dx.doi.org/10.1097/PEC.0b013e3181e5bf34

[9] Auerbach, M., Kessler, D. and Foltin, J.C. (2011) Repetitive Pediatric Simulation Resuscitation Training. Pediatric Emergency Care, 27, 29-31. http://dx.doi.org/10.1097/PEC.0b013e3182043f3b

[10] Trainor, J.L. and Krug, S.E. (2000) The Training of Pediatric Residents in the Care of Acutely Ill and Injured Children. Archives of Pediatrics and Adolescent Medicine, 154, 1154-1159.

[11] Gass, D.A. and Curry, L. (1983) Physicians’ and Nurses’ Retention of Knowledge and Skill after Training in Cardiopulmonary Resuscitation. Canadian Medical Association Journal, 128, 550-551.

[12] Andreatta, P., Saxton, E., Thompson, M. and Annich, G. (2011) Simulation-Based Mock Codes Significantly Correlate with Improved Pediatric Patient Cardiopulmonary Arrest Survival Rates. Pediatric Critical Care Medicine, 12, 33-38. http://dx.doi.org/10.1097/PCC.0b013e3181e89270 


\section{Appendix 1}

\section{Self-Assessment Questionnaire}

Gender Male/Female

Year of residency $\begin{array}{lllll}1 & 2 & 3 & 4 & 5\end{array}$

Have you completed a rotation in the PICU? Yes/No

Have you completed a PALS course in the past 2 years? Yes/No

How many pediatric resuscitations have you taken part in? 0/1 - 2/3 - 5/6 - 9/10 or more

\section{Rate You Level of Agreement with the Following Sentences}

I have good knowledge on how to manage a resuscitation requiring airway management

I feel comfortable running a resuscitation requiring airway management

I have good knowledge on how to manage a resuscitation requiring fluid resuscitation

I feel comfortable running a resuscitation of shock requiring fluid resuscitation

I am comfortable performing bag-mask ventilation

I am comfortable performing endotracheal intubation

I feel comfortable managing a pediatric resuscitation
Strongly disagree disagree neutral agree strongly agree

$\begin{array}{lllll}1 & 2 & 3 & 4 & 5 \\ 1 & 2 & 3 & 4 & 5 \\ 1 & 2 & 3 & 4 & 5 \\ 1 & 2 & 3 & 4 & 5 \\ 1 & 2 & 3 & 4 & 5 \\ 1 & 2 & 3 & 4 & 5 \\ 1 & 2 & 3 & 4 & 5\end{array}$

